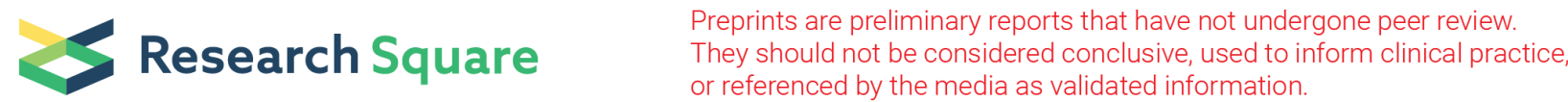

\section{Application of Real-Time Simultaneous \\ Amplification and Testing Method to Accurately and Rapidly Detect Extra-pulmonary Tuberculosis}

\section{Tongxin Li}

Chongqing Public Health Medical Center

Tao Shi ( $\nabla$ shitaostone@163.com )

The Third Hospital Affiliated Chongqing Medical University

\section{Ying Sun}

Tianjin Hexi Hospital

\section{Kan Zhou}

Chongqing Public Health Medical Center

\section{Zhenggu Huang}

Chongqing Public Health Medical Center

\section{Pengsen Wang}

Chongqing Public Health Medical Center

\section{Ming Luo}

Chongqing Public Health Medical Center

\section{Xiaoping Nie}

Chongqing Public Health Medical Center

\section{Guoqing Yang}

Chongqing Public Health Medical Center

\section{Yu Chen}

Chongqing Public Health Medical Center

\section{Yaokai Chen}

Chongqing Public Health Medical Center

\section{Research article}

Keywords: extra-pulmonary; Mycobacterium tuberculosis; simultaneous amplification and testing; specificity; sensitivity.

Posted Date: March 12th, 2020

DOI: https://doi.org/10.21203/rs.2.22566/v2 
License: (c) (i) This work is licensed under a Creative Commons Attribution 4.0 International License. Read Full License

Version of Record: A version of this preprint was published at BMC Infectious Diseases on April 22nd, 2020. See the published version at https://doi.org/10.1186/s12879-020-05036-0. 


\section{Abstract}

Background: This study aimed to establish and evaluate a simultaneous amplification and testing method for detection of extra-pulmonary tuberculosis. Methods: From January 2016 and December 2017 the pus or surgical excision from the lesions of inpatients admitted from Chongqing Public Health Treatment Center were collected. According to the clinical diagnosis, the samples were divided into two groups including extra-pulmonary tuberculosis (Group A) and other diseases excluded from tuberculosis diseases (Group B). Simultaneous detection of Mycobacterium tuberculosis (MTB) used Roche culture method, liquid culture method and simultaneous amplification and testing method (SAT). The sensitivity and specificity of the SAT method were compared with culture methods and clinical diagnosis of extrapulmonary tuberculosis. Results: For 433 extra-pulmonary tuberculosis (EPTB) specimens and 49 non-TB specimens, the SAT-TB results correlated with $80.5 \%$ (388/482 specimens) of the culture assay results. The sensitivity, specificity, and positive and negative predictive values of the SAT-TB test for the diagnosis of EPTB were $83.6 \%, 79.4 \%, 59.4 \%$, and $93.0 \%$, respectively, compared to culture methods. Compared with the clinical diagnosis of patients, the sensitivity and specificity of the SAT-TB test were $41.6 \%$ and $100 \%$, respectively, the cultures test were $29.3 \%$ and $98.0 \%$. Conclusions: SAT test is a simple and rapid test with high specificity which may enhance the detection of EPTB. SAT-TB is a higher clinical diagnosis value for EPTB in clinical microbiology laboratories.

\section{Introduction}

Tuberculosis (TB) is a chronic infectious disease that endangers human health for a long time. Its clinical manifestations are extremely diverse, with $80 \%$ of TB infections occurring in the lungs and other sites like cervical lymphadenitis, meninges, peritoneum, intestines, skin, and bones [1]. According to the WHO Global Tuberculosis Report 2015 32,000 cases of extra-pulmonary tuberculosis (EPTB) were diagnosed in nearly 530,000 new cases in China in 2014 [2]. In 2018 there were an estimated 10.0 million people fell with TB and 1.2 million death [3]. It is difficult to diagnose EPTB due to its extensive lesions and complicated clinical manifestations.

Rapid and accurate laboratory testing technology plays an important role in assisting the early diagnosis of EPTB in clinical practice. Simultaneous Amplification and Testing (SAT) is one of the molecular biological detection methods used by the tuberculosis laboratory in China in recent years. It is mainly used to detect MTB in sputum to assist diagnosis [4-7]. However, SAT has rarely been used to test EPTB specimens and therefore, its sensitivity and specificity for EPTB diagnosis are still not clear. This study aimed to evaluate the clinical accuracy of SAT in the diagnosis of EPTB by comparing sensitivity, specificity, positive predictive value and negative predictive value of both SAT and culture methods in detecting EPTB specimens.

\section{Methods}

\subsection{Patients}


482 cases of inpatients with EPTB, who were hospitalized in Chongqing Public Health and Medical Center from January 2016 to December 2017, were included in this study. Among them, there were 433 cases of confirmed EPTB patients (Group A) and 49 cases of non-TB patients (Group B). Of all the 482 patients, 259 were male and 223 were female. The average age of all 482 patients was 35.5 years, ranging from 7 years to 79 years.

\subsection{Specimen collection and pretreatment}

Specimens (pus and excised tissues) were collected during surgical procedures and stored at low temperature $\left(4-8^{\circ} \mathrm{C}\right)$. Then, the specimens were decontaminated using the $\mathrm{N}$-acetyl-L-cysteine (NALC)$\mathrm{NaOH}$ method [8]. The processed sediment was washed using a sterile $0.9 \% \mathrm{NaCl}$ solution, re-suspended in $1.5 \mathrm{ml}$ sterile $0.9 \% \mathrm{NaCl}$ solution and then equally divided into three thirds.

\subsection{Specimen separation and culture}

One of the three thirds was then centrifuged and the sediment was inoculated in both Bactec MGIT 960 system (Bacton Dickinson and Company, MD) and neutral Roche medium. The culture was regarded as positive if one or both of the above two culture methods produced positive results.

\subsection{Specimen TB-SAT detection}

Another third was centrifuged at $13000 \mathrm{rpm}$ for $5 \mathrm{~min}$, and the supernatant were discarded. The sediment was washed by $1 \mathrm{ml}$ sterile saline, and 50ul TB lysate buffer was added for re-suspension. The resuspended solution was treated by ultrasonic (power $300 \mathrm{~W}$ ) for $15 \mathrm{~min}$. 2 ul of the treated solution was added into a micro reaction tube containing $30 \mathrm{ul}$ of amplification detection solution. The micro reaction tube was placed on a dry heat thermostat $\left(60^{\circ} \mathrm{C}\right)$ for $10 \mathrm{~min}$, and then placed at $42^{\circ} \mathrm{C}$ for $5 \mathrm{~min}$, with the reaction enzyme being preheated to $42^{\circ} \mathrm{C}$ at the same time. Finally, $10 \mathrm{ul}$ of enzyme was added to each reaction tube, was quickly mixed and transferred to a real-time fluorescence quantitative PCR instrument. Reaction procedure: The fluorescence channel was set to FAM, and each cycle was at $42^{\circ} \mathrm{C}$ for $1 \mathrm{~min}$, with a total of 40 cycles; the fluorescence signal was collected once per minute, and 40 times as a total. Positive result criterion: The abscissa reading (detection time) of the intersection of the sample curve and the threshold line is $<40 \mathrm{~min}$. Negative and positive controls must be done for each test.

\subsection{MTB identification}

MTB identification was performed if the results of culture and TB-SAT were inconsistent. Take the last third re-suspended solution and perform DNA extraction and identification of MTB complexes.

\subsection{Mycobacterial species identification}

Mycobacterial species identification was performed for culture-positive but TB-SAT-negative samples according to the mycobacterial species identification test kit instructions. The reverse dot hybridization method was used to identify mycobacteria species. The reference strain of MTB (H37Rv) was obtained 
from the National Tuberculosis Reference Laboratory of the Chinese Center for Disease Control and Prevention.

\subsection{Statistical analysis}

SPSS 13.0 analysis software was used for statistical analysis. The sensitivity, specificity, positive predictive value and negative predictive value of the SAT method were compared with the culture method and clinical diagnosis of the patient. The positive rate of MTB in the culture method and SAT test was compared with Chi-square test. $P<0.05$ was considered statistically significant.

\section{Results}

\subsection{Comparison of TB-SAT and culture methods}

482 extra-pulmonary tuberculosis specimens tested by the TB-SAT and culture methods. The results were displayed in the Table 1. The positive rate of the culture methods was $26.6 \%(128 / 482)$ and the positive rate of TB-SAT was $37.3 \%$ (180/482). The positive rate of culture methods and TB-SAT were $29.3 \%$ (127/433) and $41.6 \%$ (180/433) in the Group A, respectively. The positive rate of the culture methods and TB-SAT were $4.1 \%(1 / 49)$ and $0 \%(0 / 49)$ in the Group B, respectively. The difference in the positive rates of TB-SAT and culture method was statistically significant $(X 2=106.11, P<0.05)$. Using culture method as the gold standard the results of sensitivity, specificity, positive predictive value and negative predictive value were displayed in the Table 1. Using clinical diagnosis as the standard, the results of sensitivity, specificity, positive predictive value and negative predictive value were displayed in the Table 2 . The difference in the positive rates of TB-SAT and clinical diagnosis was statistically significant $(\chi 2=27.67$, $P<0.05)$ and the positive rates of culture method and clinical diagnosis was statistically significant $(x 2=13.06, P<0.05)$.

\subsection{MTB identification results}

Among the 482 samples, results of 94 culture method were inconsistent with the results of the TB-SAT test. Of 94 samples, 73 samples were positive TB-SAT test results and negative culture results; 21 samples were negative TB-SAT test results and positive culture results. MTB identification was used on all the 94 strains. Of 73 samples the results of MTB identification were 70 positive results and 3 negative results; of the rest 21 samples the results of MTB identification were 3 positive results and 18 negative results. The details of results were displayed in the Table 3.

\subsection{Identification of mycobacteria species}

21 strains with negative TB-SAT results and positive culture results were identified. Mycobacterial species identification results showed that 3 cases were Mycobacteria Intracellular, 1 case was Mycobacterium Cheloniae Abscess Subspecies and 1 case was Mycobacterium Gordon's. The remaining 16 strains were MTB. The details were shown in Table 4. 


\section{Discussion}

Simultaneous Amplification and Testing (SAT) is a nucleic acid detection technology. The nucleic acid isothermal amplification and real-time fluorescence detection are combined in this technology. The principle is that MTB 16S rRNA was reverse transcribed using Moloney murine leukemia virus (M-MLV) reverse transcriptase in order to generate a 170-bp DNA fragment using a pair of specific primers incorporating the T7 promoter sequence in the sense primer. The DNA was transcribed to RNA by using T7 RNA polymerase to undergo successive cycles of amplification. An internal labeled probe was included and released a fluorescence signal when hybridized with the target RNA. The dynamic measurements of the real-time amplification fluorescence signal were detected using a real-time PCR instrument. This technology has been widely used in the clinical detection of MTB in sputum for the early diagnosis of tuberculosis, but the clinical value for the detection of EPTB is rarely reported.

Culture of M. tuberculosis is the gold standard for diagnosis of active MTB from clinical samples. In this study the Roche method, Bactec MGIT 960 liquid culture method and SAT method were used simultaneously to detect MTB of EPTB specimens. Using the culture methods as the gold standard, the sensitivity and specificity of TB-SAT detection were $83.6 \%$ and $79.4 \%$, respectively, and the agreement of the two methods was $80.5 \%$. Most literature reports SAT applied to the detection of MTB sensitivity in sputum in $21.2 \%-75.8 \%$ and specificity in $94.73 \%-100 \%$ [4-7,9]. Fan Lin et al. believed that the differences are related to the treatment of sputum specimens, transport and focal pulmonary tuberculosis, not with the method itself. Using clinical diagnosis as the gold standard, the sensitivity and specificity of the TBSAT detection were $41.6 \%$ and $100 \%$ in our study, respectively, which was slightly higher than the culture methods whose sensitivity and specificity were $29.3 \%$ and $98.0 \%$, respectively. This was mainly due to the positive results of SAT detection could exclude single infection of non-tuberculosis mycobacterium (NTM). However, SAT could not detect mixed infection of MTB and NTM in the same sample.

Additionally, the detection target of SAT-TB method is rRNA. RNA products are much more labile outside the reaction tube than DNA amplification products. Base on the higher specificity of SAT test that can generally be completed within 2 hours, when both the SAT and sputum smears are positive, it is most likely for the patient to be infected with the MTB. Although, in this study, the negative predictive value of SAT was low, suggesting that clinicians cannot exclude tuberculosis based on the negative SAT test result and still need to use other test results to confirm the diagnosis.

Roche culture method is a traditional and classical laboratory method in the detection of mycobacteria and has long been considered as the gold standard for the diagnosis of tuberculosis, and its test results are usually used as the gold standard for evaluating new MTB detection reagents. Taking into account the mycobacterium vitality and Roche's nutrient status of roche medium, this study used Roche culture method and Bactec MGIT 960 liquid culture method to detect specimens at the same time in order to improve the sensitivity of the culture method detection results. Bactec MGIT 960 liquid culture method was contrast with SAT test and Roche culture method was used identified the samples with discordant results between the Bactec MGIT 960 liquid culture and SAT-TB methods. The real-time fluorescent PCR method has high sensitivity and specificity for detection of MTB nucleic acid. When the results of SAT 
detection and culture method are inconsistent, another real-time fluorescent PCR detection reagent is used for evaluation. There was also a large cohort study to further analyze the specificity of the SAT detection method [10]. When the test results are inconsistent, a repeat test of SAT is performed with a detection sensitivity of $75.8 \%$. In this study, 94 specimens with inconsistent TB-SAT and culture methods were tested for MTB identification. The agreement between TB-SAT and real-time fluorescence PCR was $92.6 \%$ (87/94). In order to confirm the consistency among the methods, 21 strains of TB-SAT-negative and culture-positive strains were tested for mycobacterial species identification in this study. The results showed that only 5 cases were NTM and the other 16 cases were MTB accounted for $76.2 \%(16 / 21)$, which means that patients with SAT-negative and culture-positive patients should not be considered clinically infected with MTB. Three cases with negative TB-SAT test, positive cultures results and positive MTB assays were analyzed for possible reasons: impurities in the samples affecting TB-SAT amplification reaction, or special components in non-sputum samples (such as pus, tissue, etc.) leads to uneven distribution of target in the sample, or less bacteria in the sample and uneven distribution of template in the lysate, etc. 73 cases with negative culture method and TB-SAT positive samples were identified by PCR. The results showed that 70 cases of TB-SAT and PCR were positive, accounting for 95.9\% (70/73). The other 3 cases were culture negative and TB-SAT positive. Samples that were negative by PCR were not confirmed by further tests. This was a deficiency of this study. In addition SAT detection was also an asset in early detection and management of PTB suspects, especially in those patients who are smear negative or sputum scarce and low cost and rapidity $[9,10]$.

As same as all detection methods, there are some limitations of SAT for detection of EPTB. The first is the pretreatment of EPTB sample that is different from PTB. Some EPTB samples need more time to be processed such as bone, lymph node and so on. The second is RNA degradation. The principle of SAT is that MTB 16S rRNA was reverse transcribed using Moloney murine leukemia virus (M-MLV) reverse transcriptase in order to generate a 170-bp DNA fragment using a pair of specific primers incorporating the T7 promoter sequence in the sense primer. The DNA was transcribed to RNA by using T7 RNA polymerase to undergo successive cycles of amplification. If large RNA degraded quickly, the result may not be right. The Third is the number of live MTB in the EPTB sample. Enough live MTB can produce a large of RNA for detection. The lack of live MTB may affect detection results. In view of the above three limitations EPTB sample should be processed quickly and correctly in order to improve the results of SAT detection.

In summary, SAT has the advantages of stable reaction, high sensitivity and specificity, simple reaction conditions, simple operation, no temperature cycling, high amplification efficiency, and short reaction time; RNA amplification products and templates are both RNA and RNA are easily degraded and laboratory contamination can be greatly reduced. SAT-TB detection is a simple, rapid, sensitive and specific tuberculosis detection method. It can be used clinically to assist in the diagnosis of EPTB and at the same time to some extent increase the positive rate of EPTB patients. SAT-TB is a higher clinical diagnosis value for EPTB in clinical microbiology laboratories. 


\section{Conclusion}

SAT test is a simple and rapid test with high specificity for the detection of EPTB. SAT-TB is a higher clinical diagnosis value for EPTB in clinical microbiology laboratories.

\section{Declarations}

\section{Acknowledgements}

We thank the patients' agreement to participate in this research.

\section{Funding}

No.

\section{Availability of supporting data}

The dataset generated and/or analyzed during the current study are not publicly available due to nondisclosure agreements with data providers, but are available from the corresponding author on reasonable request.

\section{Authors' contributions}

Conceived and designed the study: TXL and TS. Collected the data: YS, KZ, ZGH, PSW, ML, XPN, GQY, YC. Analyzed the data: TXL and TS. Wrote the paper: TS. Interpreted the results: TXL, TS, ZQ and YKC. TXL. All authors have read and approved the final manuscript.

\section{Ethics approval and consent to participate}

Informed consent was obtained and had been written from all patients and the parents/legally authorized representatives of participants. The study protocol was approved by the Ethics Committee of Chongqing Public Health Medical Center of China. All methods were performed in accordance with the relevant guidelines and regulations.

\section{Consent for publication}

Not applicable.

\section{Competing interests}

The authors declare that they have no competing interests.

\section{Author details}


${ }^{1}$ Central Laboratory, Chongqing Public Health Medical Center, No. 109, Baoyu Road, Shapingba District, Chongqing 400036, China. ${ }^{2}$ Department of Orthopedics, Tianjin First Center Hospital, No.24, Fukang Road, Nankai District, Tianjin 300192, China. ${ }^{3}$ Department of Respiratory, Tianjin Hexi Hospital, No. 40, Qiongzhou Road, Hexi District, Tianjin 300202, China. ${ }^{4}$ Department of clinical laboratory, Chongqing Public Health Medical Center, No. 109, Baoyu Road, Shapingba District, Chongqing 400036, China. ${ }^{5}$ Department of Orthopedics, Chongqing Public Health Medical Center, No. 109, Baoyu Road, Shapingba District, Chongqing 400036, China. ${ }^{6}$ Medical records Room, Chongqing Public Health Medical Center, No. 109, Baoyu Road, Shapingba District, Chongqing 400036, China.

\section{References}

1. World Health Organization (WHO). Global tuberculosis report 2012. Geneva: World Health Organization; 2012.

2. World Health Organization (WHO). Global tuberculosis report 2015. Geneva: World Health. Organization; 2015.

3. World Health Organization (WHO). Global tuberculosis report 2019. Geneva: World Health Organization;2019.

4. Cui Z,Wang $Y$, Fang L, Zheng R, Huang $X$, Liu $X$, et al. Novel real-time simultaneous amplification andtesting method to accurately and rapidly detect Mycobacterium tuberculosis $\mathrm{J}$ Clin Microbiol. 2012邓50(3) $\varangle 646-650$.

5. Chen Y, Zhang L, Hong L, Luo X, Chen J, Tang L, et al. Rapid diagnosis of pulmonary tuberculosis and detection of drug resistance by combined simultaneous amplification testing and reverse dot blot. J Clin Pathol.2018; 71(6): 498-503.

6. Zhang Q, Zhou C. Comparison of laboratorytestingmethods for the diagnosis of tuberculous pleurisy in China. Sci Rep. 2017; 7(1): 45-49.

7. Yan L, Zhang Q, Xiao H. Clinical diagnostic value of simultaneous amplificationandtesting for the diagnosis of sputum-scarce pulmonary tuberculosis. BMC Infect Dis. 2017; 17(1): 545.

8. Pfyffer GE, Brown-Elliot BA \& Wallace RJ (2003). General characteristics, isolation, and staining procedures. Washington, DC, USA.

9. Yan L, Tang S, Yang Y, Shi X, Ge Y, Sun W, et al. A Large Cohort Study on the Clinical Value of Simultaneous Amplification and Testing for the Diagnosis of Pulmonary Tuberculosis. Medicine (Baltimore). 2016; 95(4): e2597.

10. Fan L, Zhang Q, Cheng L, Liu Z, Ji X, Cui Z, et al. Clinical diagnostic performance of the simultaneous amplification and testing methods for detection of the Mycobacterium tuberculosis complex for smear-negative or sputum-scarce pulmonary tuberculosis in China. Chin Med J (Engl). 2014; 127(10): 1863-7.

\section{Tables}


Table 1 Performances of SAT-TB and culture methods with clinical specimens $(n=482)$

\begin{tabular}{|c|c|c|c|c|c|c|}
\hline \multirow[b]{2}{*}{$\begin{array}{l}\text { SAT-TB } \\
\text { result }\end{array}$} & \multicolumn{2}{|c|}{$\begin{array}{c}\text { No. of specimens with culture } \\
\text { results }\end{array}$} & \multicolumn{4}{|c|}{ Mean \% } \\
\hline & Positive & Negative & Sensitivity & Specificity & PPV & NPV \\
\hline All samples & & & $83.6 \%$ & $79.4 \%$ & $59.4 \%$ & $93.0 \%$ \\
\hline Positive & 107 & 73 & & & & \\
\hline Negative & 21 & 281 & & & & \\
\hline Group A & & & $84.3 \%$ & $76.1 \%$ & $59.4 \%$ & $92.1 \%$ \\
\hline Positive & 107 & 73 & & & & \\
\hline Negative & 20 & 233 & & & & \\
\hline Group B & & & $0 \%$ & $100 \%$ & $100 \%$ & $98.0 \%$ \\
\hline Positive & 0 & 0 & & & & \\
\hline Negative & 1 & 48 & & & & \\
\hline
\end{tabular}

Table 2 Performances of SAT-TB and culture methods compared with clinical assessment of patients $(n=482)$ 


\begin{tabular}{|c|c|c|c|c|c|c|c|}
\hline \multirow[b]{2}{*}{ Method } & \multirow[b]{2}{*}{ Result } & \multicolumn{2}{|c|}{$\begin{array}{l}\text { No. of cases with } \\
\text { clinical diagnosis of TB }\end{array}$} & \multicolumn{4}{|c|}{ Mean \% } \\
\hline & & Positive & Negative & Sensitivity & Specificity & PPV & NPV \\
\hline \multirow{4}{*}{$\begin{array}{c}\text { SAT-TB } \\
\text { assay }\end{array}$} & & & & $41.6 \%$ & $100 \%$ & $100 \%$ & $16.2 \%$ \\
\hline & & & & & & & \\
\hline & Positive & 180 & 0 & & & & \\
\hline & Negative & 253 & 49 & & & & \\
\hline \multirow{3}{*}{$\begin{array}{l}\text { Culture } \\
\text { methods }\end{array}$} & & & & $29.3 \%$ & $98.0 \%$ & $99.2 \%$ & $13.6 \%$ \\
\hline & Positive & 127 & 1 & & & & \\
\hline & Negative & 306 & 48 & & & & \\
\hline
\end{tabular}

Table3 Results of MTB identification of samples that TB-SAT results were inconsistent with culture methods $(n=94)$

\begin{tabular}{ccccc}
\hline Groups & No. of samples & \multicolumn{3}{c}{ Results } \\
\cline { 2 - 5 } & & Culture methods & TB-SAT & MTB \\
\hline Group A & 17 & + & - & + \\
& 70 & - & + & + \\
Group B & 3 & - & + & - \\
& 1 & + & - & - \\
“+" Presents “Positive” “ “_" Presents “Negative”
\end{tabular}


Table 4 Results of mycobacterium species identification $(n=21)$

\begin{tabular}{|c|c|c|c|c|c|}
\hline \multirow[t]{2}{*}{ Groups } & \multirow[t]{2}{*}{ No. of samples } & \multicolumn{4}{|r|}{ Results } \\
\hline & & Culture methods & TB-SAT & MTB & Species identification \\
\hline \multirow{5}{*}{ Group A } & 3 & + & - & + & MTB \\
\hline & 13 & + & - & - & MTB \\
\hline & 2 & + & - & - & Intracellular mycobacterium \\
\hline & 1 & + & - & - & Gordon's mycobacterium \\
\hline & 1 & + & - & - & Mycobacterium cheloniae abscess subspecies \\
\hline Group B & 1 & + & - & - & Intracellular mycobacterium \\
\hline
\end{tabular}

“+” Presents “Positive” “_" Presents “Negative” 